\title{
Hořava gravity after GW170817
}

\author{
A. Emir Gümrükçüoğlu, ${ }^{1}$ Mehdi Saravani, ${ }^{2}$ and Thomas P. Sotiriou ${ }^{2,3}$ \\ ${ }^{1}$ Institute of Cosmology and Gravitation, University of Portsmouth, \\ Portsmouth PO1 3FX, United Kingdom \\ ${ }^{2}$ School of Mathematical Sciences, University of Nottingham, University Park, \\ Nottingham, NG7 2RD, United Kingdom \\ ${ }^{3}$ School of Physics and Astronomy, University of Nottingham, University Park, \\ Nottingham, NG7 2RD, United Kingdom
}

(Received 30 November 2017; published 24 January 2018)

\begin{abstract}
The recent detection of gravitational wave GW170817 has placed a severe bound on the deviation of the speed of gravitational waves from the speed of light. We explore the consequences of this detection for Hořava gravity.
\end{abstract}

DOI: 10.1103/PhysRevD.97.024032

\section{INTRODUCTION}

Hořava gravity [1] has been proposed as an ultraviolet (UV) complete theory for the gravitational interaction. The improved behavior at high energies is due to the presence of higher-order terms in spatial derivatives and this requires violations of local Lorentz invariance. The action of Hořava gravity is $[1,2]$

$$
\begin{aligned}
S= & \frac{M_{p}^{2}}{2} \int N d t \sqrt{h} d^{3} x\left(K_{i j} K^{i j}-\lambda K^{2}+\xi^{(3)} R+\eta a_{i} a^{i}\right. \\
& \left.+\frac{1}{M_{\star}^{2}} \mathcal{L}_{4}+\frac{1}{M_{\star}^{4}} \mathcal{L}_{6}\right) .
\end{aligned}
$$

$N, N^{i}$, and $g_{i j}$ are the lapse function, the shift and the induced metric on a given spacetime foliation by spacelike hypersurfaces, while $K_{i j}$ is their extrinsic curvature. $\mathcal{L}_{4}$ and $\mathcal{L}_{6}$ contain terms that are fourth-order and sixth-order in spatial derivatives, respectively. Hence, they contribute fourth and sixth powers of momenta in the dispersion relations. These contributions are suppressed at momenta below some scale $M_{*}$, where the theory becomes effectively an infrared (IR) modification of general relativity (GR), but dominate in the UV and are expected to render interactions renormalizable. The action is not invariant under the full group of diffeomorphisms, but only under diffeomorphisms that respect the foliation. This underscores that the foliation is preferred.

The first line of Eq. (1) can be thought of as the IR limit of Hořava gravity. Elevating the space-time symmetry to full diffeomorphisms by introducing a Stückelberg field $\phi$, one can write the IR action as [3]

$$
\begin{aligned}
S_{\mathrm{IR}}= & \frac{M_{Æ}^{2}}{2} \int \sqrt{-g} d^{4} x\left[{ }^{(4)} R+\alpha u^{\mu} u^{\nu} \nabla_{\mu} u_{\alpha} \nabla_{\nu} u^{\alpha}\right. \\
& \left.-\beta \nabla_{\mu} u_{\nu} \nabla^{\nu} u^{\mu}-\gamma\left(\nabla_{\mu} u^{\mu}\right)^{2}\right],
\end{aligned}
$$

where we defined $u_{\mu} \equiv-\nabla_{\mu} \phi / \sqrt{-\nabla^{\nu} \phi \nabla_{\nu} \phi}$. This action coincides with the first line of action (1) after the partial gauge fixing $\phi=t$ and with the following correspondence of parameters through

$$
\begin{array}{ll}
\lambda=\frac{1+\gamma}{1-\beta}, & \eta=\frac{\alpha}{1-\beta}, \\
\xi=\frac{1}{1-\beta}, & M_{p}^{2}=(1-\beta) M_{Æ}^{2} .
\end{array}
$$

Moreover, the covariant formulation is equivalent to a restricted version of the Einstein-Æther theory [4], in which the æether is forced to be hypersurface-orthogonal at the level of the action (i.e., before the variation) [5]. The correspondence of parameters is given in Appendix A.

Hořava gravity propagates two tensor and one scalar polarization. All three polarizations satisfy higher-order dispersion relations, as mentioned earlier. Their speeds in the infrared limit are

$$
c_{T}^{2}=\frac{1}{1-\beta}, \quad c_{S}^{2}=\frac{(2-\alpha)(\gamma+\beta)}{\alpha(1-\beta)(2+3 \gamma+\beta)} .
$$

The recent detection of a binary neutron star merger with coincident gamma ray emission has introduced remarkably strong constraints on $c_{T}$ [6]. The purpose of this brief note is to discuss the implications of this constraint for Horava gravity and to clarify how this constraint can be effectively combined with existing ones. It has recently been pointed out in Ref. [7] that the speed of the scalar polarization is almost unconstrained and our results highlight that this feature persists even after GW170817.

It is worth pointing out that our focus is on infrared viability. Hence, the higher order terms contained in $\mathcal{L}_{4}$ and $\mathcal{L}_{6}$ in action (1) will not be relevant and we will not attempt 
to give them explicitly. We will, however, discuss constraints on $M_{\star}$ and mention how theoretical considerations related to the UV properties of the theory can restrict $M_{\star}$. We will also not consider any version of Hořava gravity where additional restrictions of the action are considered in order to reduce the numbers of independent couplings, e.g., [8-12]. Even though some of these restricted models have been shown to have interesting properties-the so-called "projectable" theory [8], for instance, has been shown to be renormalizable beyond power-counting $[13]^{1}$ - they also tend to suffer from infrared viability issues [16-22].

\section{DIRECT CONSTRAINTS AND BOUNDS}

In this section we list all of the available constraints in terms of the parameters $(\alpha, \beta, \gamma)$ of action (2).

(1) Unitarity. The kinetic term for the scalar mode should have the same sign as the kinetic term of the tensor modes in order for the Hamiltonian to be bounded for linearized perturbations around flat space. This yields [2]:

$$
\frac{2+3 \gamma+\beta}{\gamma+\beta}>0
$$

(2) Perturbative stability. The coefficients of the gradient terms should have the right sign for stability [2]:

$$
0<\alpha<2, \quad \beta<1 .
$$

This condition, along with the previous one ensures that $c_{T}^{2}$ and $c_{S}^{2}$ are always positive.

(3) BBN. Cosmology provides further constraints. On a cosmological background, the effective gravitational constant that appears in the Friedmann equation is [2]

$$
G_{C}=\frac{1}{4 \pi M_{Æ}^{2}(2+3 \gamma+\beta)},
$$

while from the Newtonian limit, one can infer [23]

$$
G_{N}=\frac{1}{8 \pi M_{Æ}^{2}(1-\alpha / 2)} .
$$

This effective gravitational constant affects the expansion rate during big bang nucleosynthesis (BBN) with respect to the standard one. As a result the primordial helium abundance is modified by $[24,25]$

$$
\Delta Y_{p}=0.08\left(\frac{G_{C}}{G_{N}}-1\right)
$$

\footnotetext{
${ }^{1}$ Projectable theory in $2+1$ dimensions [14] has actually been shown to be asymptotically free [15].
}

Using the current bound $\left|\Delta Y_{p}\right|<0.01$ (99.7\% C.L.) [26-28] we obtain the following constraint

$$
\left|\frac{\alpha+3 \gamma+\beta}{2+3 \gamma+\beta}\right|<\frac{1}{8}
$$

(4) Vacuum Cherenkov bounds. Photons could decay into spin-2 or spin-0 modes in vacuum when Lorentz symmetry is violated. Cosmic rays provide a lower bound on the speed of gravitational polarizations [29]. Specific constraints for Einstein-Æther theory have been derived in Ref. [30] and they exclude subluminal propagation to very high precision. ${ }^{2}$ There is no detailed calculation for Hořava gravity, or a quantitative translation of the Einstein-Æther results (given the similarity of the theories). However, the conservative expectation is that subluminal propagation is excluded to very high accuracy for both tensor and scalar polarizations.

The absence of gravitational Cherenkov radiation can, in principle, give a bound on $M_{*}$, the scale that suppressed the higher-order corrections to the dispersion relation of gravitational waves [31,32]. However, to obtain a noteworthy bound one needs to assume that the coefficient of $p^{4}$ term (where $p$ is momentum) in the dispersion relation is negative. Moreover, such a constraint would only be trustworthy if $M_{*}$ is much bigger than the cosmic ray energies $\left(\sim 10^{11} \mathrm{GeV}\right)$. Otherwise, one would need to include the $p^{6}$ term in the analysis as well, which is expected to have a positive sign. We will not consider this type of bound below.

(5) $\mathrm{ppN}$ constraints. The two parametrized postNewtonian $(\mathrm{ppN})$ parameters which quantify preferred-frame effects are constrained by [33]

$$
\begin{aligned}
& \left|\alpha_{1}\right|<10^{-4}, \\
& \left|\alpha_{2}\right|<4 \times 10^{-7} .
\end{aligned}
$$

Using the weak field expressions for Hořava gravity $[3,34]$, these constraints translate into

$$
\begin{aligned}
&\left|\frac{4(\alpha-2 \beta)}{1-\beta}\right| \lesssim 10^{-4}, \\
&\left|\left(\frac{\alpha-2 \beta}{2-\alpha}\right)\left(1-\frac{(\alpha-2 \beta)(1+\beta+2 \gamma)}{(1-\beta)(\beta+\gamma)}\right)\right| \lesssim 10^{-7} .
\end{aligned}
$$

(6) Binary pulsars. The presence of a scalar polarization can lead to dipolar emission and this would

\footnotetext{
${ }^{2}$ There is no compelling reason to a priori exclude superluminal propagation, unlike what seems to be suggested in Ref. [30]. Hence, vacuum Cherenkov constraints are one-sided.
} 
affect the dynamics of binary pulsars. The corresponding bounds are discussed in details in Ref. [35]. Figures 1(b) and 8 of Ref. [35] present the constraints on the $\alpha=2 \beta$ plane. We will not reproduce these figures here. On this plane the ppN parameters $\alpha_{1}$ and $\alpha_{2}$ vanish but, as will be discussed below, considering this plane is no longer well motivated.

(7) Black holes. The structure of isolated black holes can, in principle, provide constraints [36-39]. However, such constraints are significantly weaker than the binary pulsar constraints (at least on the $\alpha=$ $2 \beta$ plane).

(8) Gravitational waves. The observation of the binary neutron star merger GW170817 with coincident gamma ray emission [6] yields

$$
-3 \times 10^{-15} \leq c_{T}-1 \leq 7 \times 10^{-16},
$$

which implies that

$$
|\beta| \lesssim 10^{-15}
$$

Gravitational wave observations also provide a very mild lower bound on $M_{\star}$ of the order of $e V[7,40]$. This bound comes from considering the effect of the $p^{4}$ term in the dispersion relation (where $p$ is momentum) that is suppressed by $M_{\star}^{2}$.

\section{THEORETICAL CONSIDERATIONS AND INDIRECT CONSTRAINTS}

The direct observational bounds obtained in the previous section affect mainly the parameters of the IR effective theory. The parameters that become relevant in the UV are very weakly affected. In particular, gravitational waves provide only a very weak bound of $M_{\star}$, as mentioned above. In principle, laboratory test of gravity at small length scales can place a direct lower bound on $M_{\star}$ (as an energy scale), but current precision would place this bound in the meV range [41], so it would be also particularly weak.

Lorentz violations in the standard model are much more tightly constrained than in gravity. Indeed, if one were to assume that $M_{\star}$ is a universal Lorentz violation scale for gravity and matter alike, then observations of the synchrotron radiation from the Crab nebula would require $M_{\star}>$ $2 \times 10^{16} \mathrm{GeV}$ [42]. However, if there is a mechanism to suppress the percolation of Lorentz symmetry breaking from gravity to matter, then there is no reason to believe that $M_{\star}$ is a universal Lorentz violation scale. Moreover, such a mechanism seems to be necessary to keep Lorentz violation at bay already for lower mass dimension operators in the standard model [43]. It has been suggested that the weak coupling between gravity and matter might suffice to suppress the percolation [44] but it is not clear how well this works in practice for Hořava gravity [45-47].
Irrespective of the details, it is intuitive that experiments will impose a lower bound on $M_{\star}$. Interestingly, theoretical considerations can yield an upper bound. This is because the IR part of Hořava gravity, or equivalently action (2) exhibits strong coupling at a certain scale $M_{\mathrm{SC}}$ [20]. In particular, derivative self-interactions of the scalar mode compromise perturbativity. Provided that $M_{\mathrm{SC}}$ is sufficiently high, strong coupling is not an issue for infrared viability. However, power-counting renormalizability for Hořava gravity has been argued on the basis of perturbativity and hence, strong coupling is a threat to the original motivation of the theory [20]. It turns out that having $M_{*}<M_{\mathrm{SC}}$-i.e., having the new physics coming from the UV completion kick in at low enough energies - can resolve the strong coupling problem [3]. However, as we will review in a bit more detail below, $M_{\mathrm{SC}}$ is controlled by the couplings of the infrared part of the action, $(\lambda, \xi, \eta)$ or $(\alpha, \beta, \gamma)$, which satisfy the bounds given in the previous section. Hence, $M_{\star}$ ends up having to satisfy an upper bound.

The $M_{\mathrm{SC}}$ can been calculated in the decoupling limit, which for action (2) corresponds to $M_{Æ}^{2} \rightarrow \infty$ while keeping $\alpha M_{Æ}^{2}, \beta M_{Æ}^{2}, \gamma M_{Æ}^{2}$ fixed. This requires $\alpha, \beta$, $\gamma \rightarrow 0$. A detailed calculation was presented in Ref. [21]. It is worth pointing out that $\beta$ has been set to zero there. This can indeed be done without loss of generality by a suitable time rescaling in action (1) or field redefinitions in action (2). We have avoided it here because the rescaling affects the various speeds. We discuss in more details how what we report corresponds to the results of Ref. [21] in Appendix B.

In the limit where $(\alpha, \beta, \gamma) \ll 1$ (consistent with the decoupling approximation), $M_{\mathrm{SC}}$ can have one of two different values depending on the magnitude of $c_{S}^{2}$, namely

$$
M_{\mathrm{SC}} \approx\left\{\begin{array}{ll}
\sqrt{\alpha} M_{Æ} c_{S}^{3 / 2} & , c_{S}^{2}<1 \\
\sqrt{\alpha} M_{Æ} c_{S}^{-1 / 2} & , c_{S}^{2}>1
\end{array} .\right.
$$

It is worth stressing that when $(\alpha, \beta, \gamma) \ll 1$, and hence in the decoupling limit as well, $M_{\rightleftarrows} \sim M_{p}$ and both scales could be taken to be equal to the gravitational coupling scale as measured by experiments.

\section{ALLOWED REGION OF THE PARAMETER SPACE}

Prior to GW170817, ppN constraints were considered particularly restrictive because, assuming that $\beta \ll 1$, they require $|\alpha-2 \beta| \lesssim 10^{-4}$. Hence, they restrict the 3-dimensional parameter space to a 2-dimensional surface with a width of $10^{-4}$. All other constraints were either one-sided or weaker, so it was common practice to impose $\alpha=2 \beta$ in order to satisfy both $\mathrm{ppN}$ bounds to infinite accuracy (modulo tunings) and present graphically existing and new constraints on the $\alpha=2 \beta$ plane, see for example 

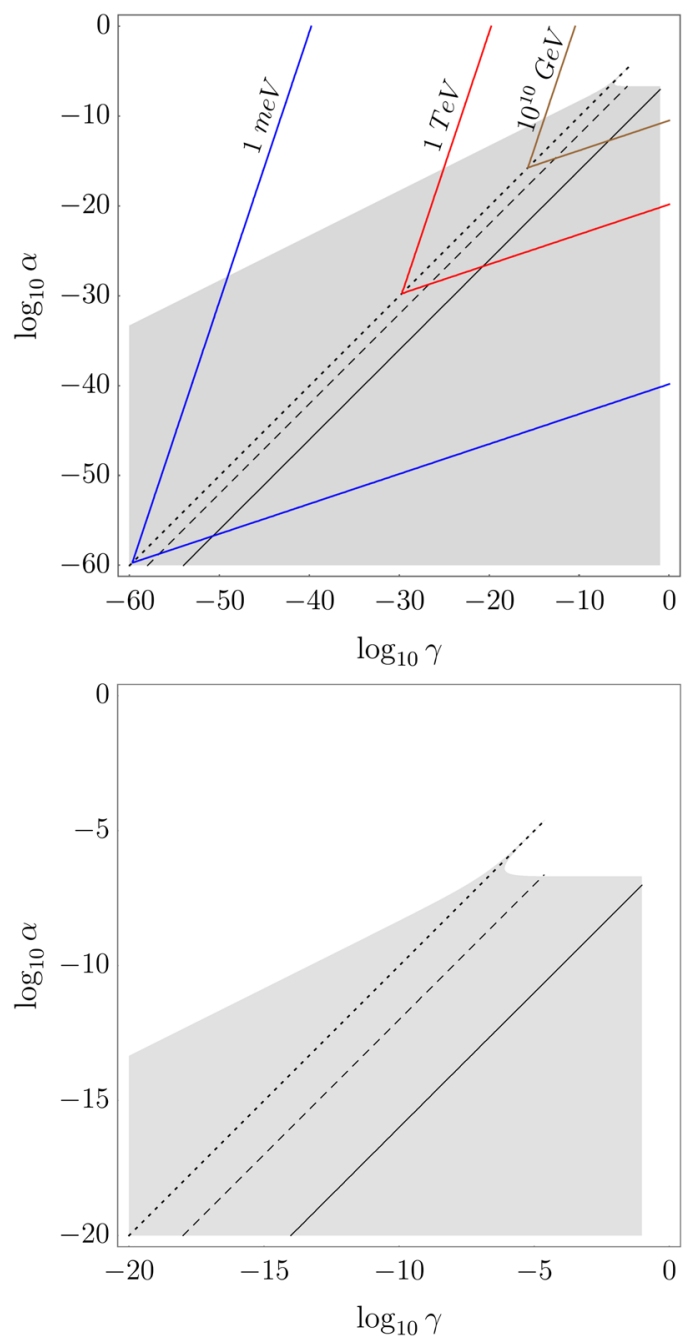

FIG. 1. The grey area shows the region of parameter space which is compatible with the constraints [Sec. II] for $\beta=0$. The dotted, dashed, and solid lines correspond to $c_{S}=1, c_{S}=10$, and $c_{S}=1000$, respectively. The blue, red, and brown lines show $M_{\mathrm{SC}}=1 \mathrm{meV}, M_{\mathrm{SC}}=1 \mathrm{TeV}$, and $M_{\mathrm{SC}}=10^{10} \mathrm{GeV}$. The lower panel focuses on the region $\alpha, \gamma>10^{-20}$.

Refs. [35,36,39]. The double-sided constraint on $c_{T}$ or $\beta$ from GW170817 changes the picture drastically. The allowed region of the $\alpha=2 \beta$ plane shrinks to a line segment with width less than $10^{-15}$, as one can always think of this plane as parametrized by $\beta$ itself and some other combination of the couplings (cf., with the figures in Refs. [35,36,39]).

Indeed it is now much more informative to focus on the $\beta=0$ plane of the parameter space or, more generally, $|\beta| \sim$ $10^{-15}$ sections, as in Figs. $1-3$. The top panel of each figure shows the whole parameter space. The blue curve corresponds to $M_{\mathrm{SC}}=1 \mathrm{meV}$. Given that $M_{*}<M_{\mathrm{SC}}$, the constraint $M_{*}>1 \mathrm{meV}$, which is derived from binary black hole mergers [32], excludes the region of parameter space corresponding to $M_{\mathrm{SC}}<1 \mathrm{meV}$. The red and brown curves correspond to $M_{\mathrm{SC}}=1 \mathrm{TeV}$ and $M_{\mathrm{SC}}=10^{10} \mathrm{GeV}$,
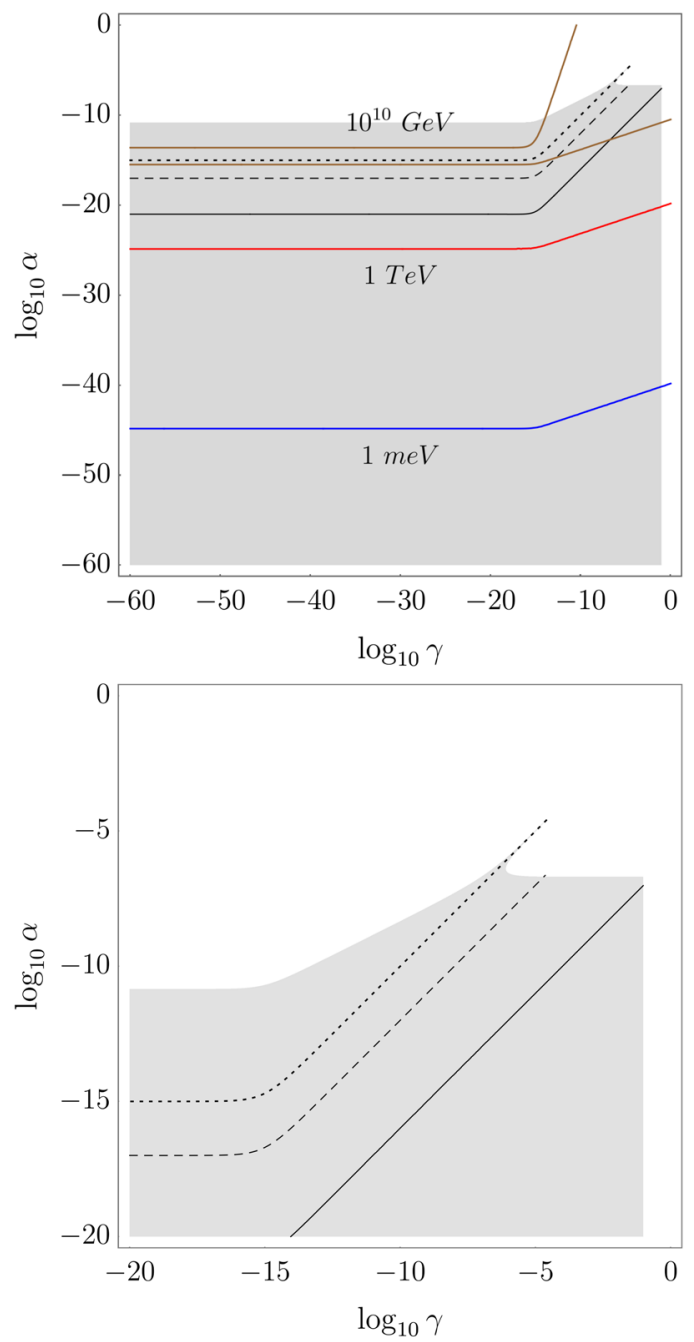

FIG. 2. Same as Fig. 1 but for $\beta=10^{-15}$.

respectively. These curves demonstrate how stricter bounds on $M_{*}$ would constraint the parameter space. The lower panels zoom on the region $\alpha, \gamma>10^{-20}$. The dotted, dashed and solid lines correspond to choice of $\alpha$ and $\gamma$ that lead to $c_{S}=1, c_{S}=10$ and $c_{S}=1000$, respectively. They have been included to highlight that the $c_{S}$ remains virtually unconstrained. It should be stressed that we have not imposed the vacuum Cherenkov constraints. Imposing them in a conservative fashion corresponds to excluding the part of the shaded region above the $c_{S}^{2}=1$ dotted line in each plot.

\section{DISCUSSION}

The detection of gravitational waves with an electromagnetic counterpart (GW170817), emitted by a binary neutron star merger, has put a stringent constraint on the speed of tensor modes. This translates to the tightest constraint so far in one of the parameters of Hořava gravity and it motivates revisiting the allowed region of the 3-dimensional parameter space. Instead of focusing on 

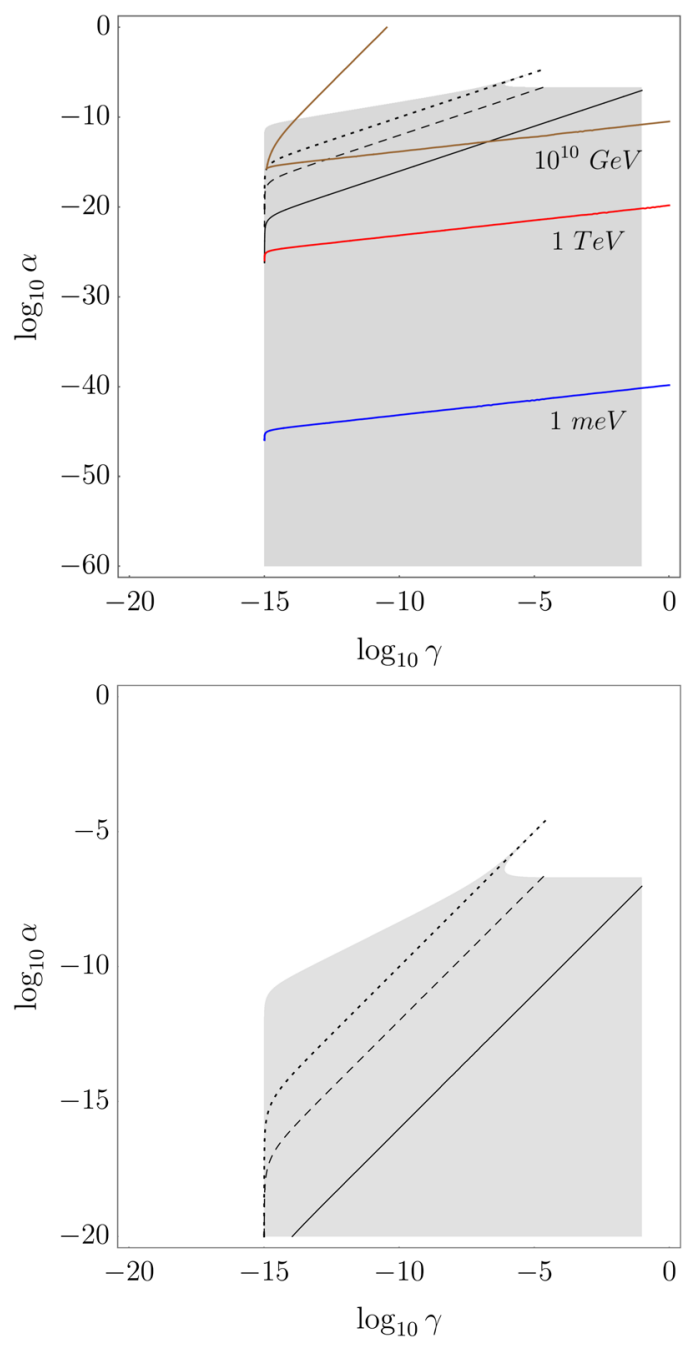

FIG. 3. Same as Fig. 1 but for $\beta=-10^{-15}$.

the 2-d plane that leads to exact agreement with general relativity in the weak field limit, as had been done in the literature so far, we focussed on 2-dimensional sections that satisfy the gravitational wave speed constraint and considered all other known constraints. The graphic representation of these constraints turns out to be quite illuminating in at least two respects:

(1) The strong coupling scale $M_{\mathrm{SC}}$ is bounded from below by the Lorentz breaking scale $M_{\star}$, as discussed extensively in the text. $M_{\star}$ is in turn bounded from below by observations that probe the higher order terms in the dispersion relation. Improving bounds on $M_{\star}$ can hence reduce the parameter space significantly or rule out Hořava gravity entirely as a perturbatively renormalizable theory of gravity.

(2) Even though the constraints on the parameters $\alpha, \beta$, and $\gamma$ are very tight, the speed of the scalar polarization remains virtually unconstrained. This stems from the type of dependence $c_{S}$ has on these parameters and, more fundamentally, from the fact that the limit to general relativity is not smooth.
Remarkably, even a very mild constraint on $c_{S}$ would rule out a vast portion of the parameter space. The above motivate finding novel ways to improve constraints on $M_{\star}$ and $c_{S}$.

The prospects of measuring or constraining $c_{S}$ and its importance in the context of Lorentz violations in gravity have been recently discussed in Ref. [7]. In the context of Hořava gravity, one could hope to obtain stricter bounds on $M_{\star}$ and $c_{S}$ by precise gravitational waveform modeling. In principle, trustworthy bounds on $M_{\star}$ could also be obtained from nongravitational experiments (along the lines of Ref. [42]), if percolations of Lorentz symmetry from gravity to matter were well understood. Both prospects are quite challenging but would significantly enhance our understanding of the role of Lorentz symmetry in gravitation.

\section{ACKNOWLEDGMENTS}

We are grateful to Andrew Coates, Ted Jacobson, and Tony Padilla for illuminating discussions. A.E. G. acknowledges financial support from the European Research Council (ERC) under the European Union's Horizon 2020 research and innovation programme (Grant No. 646702 CosTesGrav). M. S. is supported by the Royal Commission for the Exhibition of 1851. T. P. S. acknowledges funding from the European Research Council under the European Union's Seventh Framework Programme (FP7/2007-2013) / ERC grant agreement no. 306425 "Challenging General Relativity" and partial support from the STFC Consolidated Grant No. ST/ P000703/1. T. P. S. would also like to acknowledge networking support by the COST Action GWverse CA16104.

\section{APPENDIX A: CORRESPONDENCE WITH HYPERSURFACE ORTHOGONAL EINSTEIN-ÆTHER THEORY}

The action for Einstein-Æther theory [4] is given by

$$
\begin{aligned}
S= & \frac{M_{\text {Е }}^{2}}{2} \int \sqrt{-g} d^{4} x\left[{ }^{(4)} R-c_{1} \nabla_{\mu} u_{\nu} \nabla^{\mu} u^{\nu}\right. \\
& -c_{2}\left(\nabla_{\mu} u^{\mu}\right)^{2}-c_{3} \nabla_{\mu} u_{\nu} \nabla^{\nu} u^{\mu} \\
& \left.+c_{4} u_{\alpha} u_{\beta} \nabla^{\alpha} u_{\mu} \nabla^{\beta} u^{\mu}\right]
\end{aligned}
$$

where the norm of the æther field is constrained by $u^{\mu} u_{\mu}=-1$. If the æther field is hypersurface orthogonal it can simply be written as the (normalized) gradient of a single scalar:

$$
u_{\mu}=-\frac{\nabla_{\mu} \phi}{\sqrt{-\nabla_{\nu} \phi \nabla^{\nu} \phi}} .
$$

When this form of the vector field is imposed at the level of the action, i.e., before the variation, the $c_{1}$ term is no longer 
independent, and can be written as a combination of the $c_{3}$ and $c_{4}$. The resulting action matches the one in Eq. (2), and the correspondence of parameters is

$$
\alpha=c_{1}+c_{4}, \quad \beta=c_{1}+c_{3}, \quad \gamma=c_{2} .
$$

\section{APPENDIX B: TIME RESCALING AND THE VALUE OF $\beta$ PARAMETER}

In action (1) one can set $\xi=1$ by performing the time rescaling

$$
d \tilde{t}=\sqrt{\xi} d t
$$

Given the correspondence of couplings in Eq. (3), in the covariant picture of action (2) this maps to $\beta=0$. Note that $\xi>0$ (or $\beta<1)$ is required for stability, see Sec. II. Time rescalings do not leave speeds invariant clearly and the specific one corresponds to choosing the time coordinate such that the speed of tensor gravitational waves is set to 1 . This becomes clearer if one tries to set $\beta=0$ directly in the covariant setup of action (2), as it requires a particular metric redefinition and a $u_{\mu}$ rescaling of the type discussed in Ref. [48]. Such redefinitions leave the action formally invariant and for the specific ones that leads to $\beta=0$ the new metric defines the null propagation cones of spin-2 gravitons. It should be emphasized that the speed of light also changes and, assuming it was 1 initially, it becomes $\sqrt{1-\beta}$ after either of the two equivalent procedures discussed above. The complete mapping of couplings is

$$
\begin{array}{ll}
\tilde{\alpha}=\alpha, & \tilde{\gamma}=\frac{\gamma+\beta}{1-\beta} \\
\tilde{\beta}=0, & \tilde{M}_{Æ}^{2}=\sqrt{1-\beta} M_{A E}^{2},
\end{array}
$$

where an overtilde denotes the new couplings.

Reference [21] performs calculations in the decoupling limit but it resorts to this limit after having set $\tilde{\beta}$ to zero as described above. Hence, the limit is taken to be $\tilde{M}_{Æ}^{2} \rightarrow \infty$ while keeping $\tilde{\alpha} \tilde{M}_{Æ}^{2}, \tilde{\gamma} \tilde{M}_{Æ}^{2}$ fixed. This implies that $\tilde{\alpha}$, $\tilde{\gamma} \rightarrow 0$. while there is no further explicit reference to $\beta$. The speed of the scalar polarization in this limit is $\tilde{c}_{\mathrm{dec}}^{2}=\tilde{\gamma} / \tilde{\alpha}$. In the process of determining the smallest suppression scale for cubic interactions, and therefore identifying the strong coupling scale, the authors of Ref. [21] impose $\tilde{c}_{\mathrm{dec}} \leq 1$, with the justification that it is preferable to avoid superluminality. Under this assumption the strong coupling scale turns out to be

$$
\tilde{M}_{\mathrm{SC}}=\sqrt{\tilde{\alpha}} \tilde{M}_{\rightleftarrows} \tilde{c}_{\mathrm{dec}}^{3 / 2},
$$

as this scale has the largest positive exponent for $\tilde{c}_{\mathrm{dec}}$. A minor points of caution is that, after the time rescaling (B1) the speed of light is no longer unity but $\sqrt{1-\beta}$. Hence, superluminal propagation in the decoupling limit corresponds to $\tilde{c}_{\mathrm{dec}}^{2}>1-\beta$. More importantly, in a Lorentz violating theory with a preferred foliation there is no pertinent reason to exclude superluminal propagation. On the contrary, the vacuum Cherenkov bounds disfavor subluminal modes.

The decoupling limit as defined in Sec. III is $M_{Æ}^{2} \rightarrow \infty$ while keeping $\alpha M_{Æ}^{2}, \beta M_{Æ}^{2}, \gamma M_{Æ}^{2}$ fixed. This requires $\alpha, \beta, \gamma \rightarrow 0$, so it does not correspond exactly to the limit taken in Ref. [21]. However, when $\beta \rightarrow 0$ there is perfect agreement. Moreover, $\tilde{c}_{\mathrm{dec}}^{2} \sim c_{S}^{2}$ and the first point of caution above becomes moot. Indeed, following the line of Ref. [21] but relaxing the assumption $\tilde{c}_{\mathrm{dec}} \leq 1$, the strong coupling scale is

$$
M_{\mathrm{SC}}= \begin{cases}\sqrt{\alpha} M_{\circledast} c_{S}^{3 / 2}(1-\beta)^{3 / 4}, & c_{S}^{2}(1-\beta)<1 \\ \sqrt{\alpha} M_{Æ} c_{S}^{-1 / 2}(1-\beta)^{-1 / 4}, & c_{S}^{2}(1-\beta)>1\end{cases}
$$

where we have purposefully not taken the limit $\beta \rightarrow 0$. These expressions agree with that of Eq. (15) when $\alpha$, $\beta, \gamma \ll 1$.
[1] P. Horava, Quantum gravity at a Lifshitz point, Phys. Rev. D 79, 084008 (2009).

[2] D. Blas, O. Pujolas, and S. Sibiryakov, Consistent Extension of Horava Gravity, Phys. Rev. Lett. 104, 181302 (2010).

[3] D. Blas, O. Pujolas, and S. Sibiryakov, Models of nonrelativistic quantum gravity: The good, the bad and the healthy, J. High Energy Phys. 04 (2011) 018.

[4] T. Jacobson and D. Mattingly, Gravity with a dynamical preferred frame, Phys. Rev. D 64, 024028 (2001).
[5] T. Jacobson, Extended Horava gravity and Einstein-aether theory, Phys. Rev. D 81, 101502 (2010); Erratum, Phys. Rev. D 82, 129901(E) (2010).

[6] B. P. Abbott et al. (LIGO Scientific and Virgo and FermiGBM and INTEGRAL Collaborations), Gravitational waves and gamma-rays from a binary neutron star merger: GW170817 and GRB 170817A, Astrophys. J. 848, L13 (2017).

[7] T. P. Sotiriou, Detecting Lorentz Violations with Gravitational Waves from Black Hole Binaries, arXiv:1709.00940 [Phys. Rev. Lett. (to be published)]. 
[8] T. P. Sotiriou, M. Visser, and S. Weinfurtner, Phenomenologically Viable Lorentz-Violating Quantum Gravity, Phys. Rev. Lett. 102, 251601 (2009).

[9] S. Weinfurtner, T. P. Sotiriou, and M. Visser, Projectable Horava-Lifshitz gravity in a nutshell, J. Phys. Conf. Ser. 222, 012054 (2010).

[10] P. Horava and C. M. Melby-Thompson, General covariance in quantum gravity at a Lifshitz point, Phys. Rev. D 82, 064027 (2010).

[11] D. Vernieri and T. P. Sotiriou, Horava-Lifshitz gravity: Detailed balance revisited, Phys. Rev. D 85, 064003 (2012).

[12] D. Vernieri and T. P. Sotiriou, Horava-Lifshitz gravity with detailed balance, J. Phys. Conf. Ser. 453, 012022 (2013).

[13] A. O. Barvinsky, D. Blas, M. Herrero-Valea, S. M. Sibiryakov, and C. F. Steinwachs, Renormalization of Horava gravity, Phys. Rev. D 93, 064022 (2016).

[14] T. P. Sotiriou, M. Visser, and S. Weinfurtner, Lowerdimensional Horava-Lifshitz gravity, Phys. Rev. D 83, 124021 (2011).

[15] A. O. Barvinsky, D. Blas, M. Herrero-Valea, S. M. Sibiryakov, and C.F. Steinwachs, Horava Gravity is Asymptotically Free (in $2+1$ Dimensions), Phys. Rev. Lett. 119, 211301 (2017).

[16] T. P. Sotiriou, M. Visser, and S. Weinfurtner, Quantum gravity without Lorentz invariance, J. High Energy Phys. 10 (2009) 033.

[17] C. Charmousis, G. Niz, A. Padilla, and P. M. Saffin, Strong coupling in Horava gravity, J. High Energy Phys. 08 (2009) 070 .

[18] D. Blas, O. Pujolas, and S. Sibiryakov, On the extra mode and inconsistency of Horava gravity, J. High Energy Phys. 10 (2009) 029.

[19] K. Koyama and F. Arroja, Pathological behaviour of the scalar graviton in Horava-Lifshitz gravity, J. High Energy Phys. 03 (2010) 061.

[20] A. Papazoglou and T. P. Sotiriou, Strong coupling in extended Horava-Lifshitz gravity, Phys. Lett. B 685, 197 (2010).

[21] I. Kimpton and A. Padilla, Lessons from the decoupling limit of Horava gravity, J. High Energy Phys. 07 (2010) 014.

[22] S. Mukohyama, Horava-Lifshitz cosmology: A review, Classical Quantum Gravity 27, 223101 (2010).

[23] T. Jacobson, Einstein-aether gravity: A status report, Proc. Sci., QG-PH2007 (2007) 020, [arXiv:0801.1547].

[24] X. 1. Chen, R. J. Scherrer, and G. Steigman, Extended quintessence and the primordial helium abundance, Phys. Rev. D 63, 123504 (2001).

[25] S. M. Carroll and E. A. Lim, Lorentz-violating vector fields slow the universe down, Phys. Rev. D 70, 123525 (2004).

[26] Y. I. Izotov, T. X. Thuan, and N. G. Guseva, A new determination of the primordial $\mathrm{He}$ abundance using the He i $\lambda 10830 \AA$ emission line: cosmological implications, Mon. Not. R. Astron. Soc. 445, 778 (2014).

[27] E. Aver, K. A. Olive, and E. D. Skillman, The effects of He I $\lambda 10830$ on helium abundance determinations, J. Cosmol. Astropart. Phys. 07 (2015) 011.

[28] C. Patrignani et al. (Particle Data Group Collaboration), Review of particle physics, Chin. Phys. C 40, 100001 (2016).

[29] G. D. Moore and A. E. Nelson, Lower bound on the propagation speed of gravity from gravitational Cherenkov radiation, J. High Energy Phys. 09 (2001) 023.
[30] J. W. Elliott, G. D. Moore, and H. Stoica, Constraining the new Aether: Gravitational Cerenkov radiation, J. High Energy Phys. 08 (2005) 066.

[31] S. Kiyota and K. Yamamoto, Constraint on modified dispersion relations for gravitational waves from gravitational Cherenkov radiation, Phys. Rev. D 92, 104036 (2015).

[32] N. Yunes, K. Yagi, and F. Pretorius, Theoretical physics implications of the binary black-hole mergers GW150914 and GW151226, Phys. Rev. D 94, 084002 (2016).

[33] C. M. Will, The confrontation between general relativity and experiment, Living Rev. Relativity 9, 3 (2006).

[34] D. Blas and H. Sanctuary, Gravitational radiation in Horava gravity, Phys. Rev. D 84, 064004 (2011).

[35] K. Yagi, D. Blas, E. Barausse, and N. Yunes, Constraints on Einstein-Æther theory and Hořava gravity from binary pulsar observations, Phys. Rev. D 89, 084067 (2014); Erratum, Phys. Rev. D 90, 069902(E) (2014); Erratum, Phys. Rev. D 90, 069901(E) (2014).

[36] E. Barausse, T. Jacobson, and T. P. Sotiriou, Black holes in Einstein-aether and Horava-Lifshitz gravity, Phys. Rev. D 83, 124043 (2011).

[37] E. Barausse and T.P. Sotiriou, A No-Go Theorem for Slowly Rotating Black Holes in Horava-Lifshitz Gravity, Phys. Rev. Lett. 109, 181101 (2012); Erratum, Phys. Rev. Lett. 110, 039902(E) (2013).

[38] E. Barausse and T. P. Sotiriou, Slowly rotating black holes in Horava-Lifshitz gravity, Phys. Rev. D 87, 087504 (2013).

[39] E. Barausse and T. P. Sotiriou, Black holes in Lorentzviolating gravity theories, Classical Quantum Gravity 30, 244010 (2013).

[40] B. P. Abbott et al. (LIGO Scientific and VIRGO Collaborations), GW170104: Observation of a 50-SolarMass Binary Black Hole Coalescence at Redshift 0.2, Phys. Rev. Lett. 118, 221101 (2017).

[41] E. G. Adelberger, J. H. Gundlach, B. R. Heckel, S. Hoedl, and S. Schlamminger, Torsion balance experiments: A low-energy frontier of particle physics, Prog. Part. Nucl. Phys. 62, 102 (2009).

[42] S. Liberati, L. Maccione, and T. P. Sotiriou, Scale Hierarchy in Horava-Lifshitz Gravity: A Strong Constraint from Synchrotron Radiation in the Crab Nebula, Phys. Rev. Lett. 109, 151602 (2012).

[43] R. Iengo, J. G. Russo, and M. Serone, Renormalization group in Lifshitz-type theories, J. High Energy Phys. 11 (2009) 020.

[44] M. Pospelov and Y. Shang, On Lorentz violation in HoravaLifshitz type theories, Phys. Rev. D 85, 105001 (2012).

[45] M. Colombo, A. E. Gumrukcuoglu, and T. P. Sotiriou, Horava gravity with mixed derivative terms, Phys. Rev. D 91, 044021 (2015).

[46] M. Colombo, A. E. Gumrukcuoglu, and T. P. Sotiriou, Horava gravity with mixed derivative terms: Power counting renormalizability with lower order dispersions, Phys. Rev. D 92, 064037 (2015).

[47] A. Coates, M. Colombo, A. E. Gumrukcuoglu, and T. P. Sotiriou, Uninvited guest in mixed derivative Hořava gravity, Phys. Rev. D 94, 084014 (2016).

[48] B. Z. Foster, Metric redefinitions in Einstein-Aether theory, Phys. Rev. D 72, 044017 (2005). 